\title{
Problemas multiplicativos envolvendo combinatória: estratégias de resolução empregadas por alunos do Ensino Fundamental público ${ }^{1}$
}

\section{Multiplicative problems including combinatorics: solving strategies adopted by Public Elementary School students}

\author{
Leny R. M. Teixeira ${ }^{2}$ \\ Edileni G. J. de Campos ${ }^{3}$ \\ Mônica Vasconcellos ${ }^{4}$ \\ Sheila Denize Guimarães ${ }^{5}$
}

1 A proposta desta pesquisa se justifica dada sua filiação a um projeto mais amplo, ligado ao GT Psicologia da Educação Matemática da ANPEPP - Associação nacional de pesquisa e pós-graduação em psicologia (2004), coordenado por Márcia Regina Ferreira de Brito (Unicamp - Universidade Estadual de Campinas). O GT propôs-se a realizar uma pesquisa coletiva envolvendo várias regiões do país, com o objetivo de fazer um levantamento das principais dificuldades que os alunos do Ensino Fundamental apresentam na resolução de problemas multiplicativos. O presente trabalho constitui um desdobramento desta pesquisa mais geral, e se circunscreveu aos dados da região Centro-Oeste, representada por alunos da cidade de Campo Grande. Apoio: FUNDECT/MS, Brasil (Fundação de Apoio ao Desenvolvimento do Ensino, Ciência e Tecnologia do Estado de Mato Grosso do Sul) .

2 Doutora em Psicologia Escolar pela Universidade de São Paulo. Professora no PPGEMestrado e Doutorado em Educação da Universidade Católica Dom Bosco do Mato Grosso do Sul (UCDB / MS), Brasil (1teixeira@stetnet.com.br).

3 Doutoranda em Educação na Universidade Federal de Mato Grosso do Sul (UFMS), Brasil. (edileni@brturbo.com.br).

4 Doutora em Educação pela Universidade Federal de Mato Grosso do Sul (UFMS). Professora na UFMS/Três Lagoas, Brasil (vasconcellosdeoliveira@hotmail.com).

5 Doutora em Educação pela Universidade Federal de Mato Grosso do Sul (UFMS). Professora na UFMS/Três Lagoas, Brasil. (sheiladgui@hotmail.com). 


\title{
RESUMO
}

Neste trabalho apresentamos os dados de uma pesquisa que teve por objetivo verificar o desempenho de alunos do $6 .^{\circ}$ ao $9 .^{\circ}$ anos do Ensino Fundamental na resolução de oito problemas multiplicativos, envolvendo raciocínio combinatório. Os problemas diferiam em relação à quantidade de variáveis e à quantidade de algarismos utilizados nos fatores. Para tanto, por meio de uma entrevista clínica levantamos as principais estratégias e erros produzidos por 40 alunos de duas escolas públicas (A e B) da cidade de Campo Grande-MS, as quais fizeram parte de uma pesquisa mais ampla realizada anteriormente. Os dados revelam que o desempenho foi melhor nos problemas com duas variáveis e com fatores de valores baixos, não havendo diferença de desempenho entre alunos do $6 .^{\circ}$ e do $9 .^{\circ}$ anos. De modo geral, as dificuldades que emergiram estavam relacionadas: 1) aos modelos intuitivos que os alunos têm a respeito da multiplicação (em especial o da soma repetida); 2) a estrutura semântica do problema; 3) as preferências numéricas quanto ao tamanho dos números, formas de representar o problema e interpretação do enunciado verbal. Por ser a multiplicação uma operação bastante complexa envolvendo, em sua resolução, processos cognitivos abstratos, acreditamos que o professor precisa conhecê-los para favorecer a aprendizagem dos alunos.

Palavras-chave: Aprendizagem matemática; problemas de combinatória; processos cognitivos; Ensino Fundamental.

\begin{abstract}
This work presents data from a study which aimed at verifying the performance of Elementary School $6^{\text {th }}$ and $9^{\text {th }}$ graders when solving eight multiplicative problems involving combinatorial thinking. The problems were different concerning the amount of variables and the amount of numerical digits used in factors. Therefore, through a clinical interview, we listed the main strategies and mistakes produced by 40 graders from two public schools (A and B) in the municipality of Campo Grande (Mato Grosso do Sul - MS - State) which were part of a larger research developed before. The observations reveal that the performance was better in problems with two variables and factors with low values. There was no alteration in the performance among the $6^{\text {th }}$ and $9^{\text {th }}$ graders. In general, the difficulties found were related to: 1) intuitive models students have when dealing with multiplication (especially the one including repeated addition); 2) the semantic structure of the problem; 3) numerical preferences regarding the quantity of numerical digits, ways of representing the problem and interpretation of verbal problem statements. Because multiplication is a very complex operation involving abstract cognitive processes in its solution, we believe that the teacher needs to know them to facilitate students' learning.
\end{abstract}


Keywords: Mathematical learning; combinatorial problems; cognitive processes; Elementary School.

É sabido que as crianças têm grandes dificuldades em aprender Matemática e que o ensino dessa disciplina na escola apresenta muitos problemas e precisa ser reformulado. Um desses problemas refere-se à tendência formal e linear do ensino dessa disciplina, que prioriza a utilização de "[...] procedimentos mecânicos, desprovidos de significados para os alunos. Há urgência em reformular objetivos, rever conteúdos e buscar metodologias compatíveis com a formação que hoje a sociedade reclama" (BRASIL, 2000, p. 15).

Um fator que não poderia deixar de ser mencionado, quando nos propomos a discutir a problemática que envolve o ensino da Matemática, é a falta de sintonia entre o que é apresentado pelos teóricos da Educação como indicadores de êxito em sala de aula e a verdadeira compreensão e o domínio desses indicadores por parte dos docentes, o que acaba comprometendo a aprendizagem dos alunos. Pavanello (2002) afirma que investigações feitas por diferentes instituições, com o propósito de avaliar o conhecimento matemático dos alunos que frequentam ou frequentaram nos últimos anos a escola básica brasileira, mostraram que os mesmos não possuem nem ao menos a compreensão dos conceitos mais simples dessa área do saber.

Uma outra pesquisa, realizada por Carnoy, Gove e Marshall (2003), apresenta os resultados de uma análise das práticas de ensino de Matemática em salas de aula do Ensino Fundamental em escolas de três países: Brasil, Chile e Cuba. Os dados apontaram que as escolas brasileiras se enquadravam na categoria de aulas menos exigentes em termos de capacidade cognitiva exigida dos alunos, obtendo a média mais baixa, tanto em demanda cognitiva, como em proficiência matemática. Essa média estava relacionada ao formato das aulas, centradas na aquisição de respostas corretas sem desenvolver a compreensão. "Grande parte das aulas brasileiras consistia na professora escrevendo no quadro-negro, os alunos copiando, com pouca interação. As explicações, quando ocorriam, limitavam-se a descrever o procedimento sendo utilizando" (CARNOY; GOVE; MARSHALL, 2003, p. 21).

Em síntese, do ponto de vista didático, algumas investigações (MANRIQUE, 2003; PAVANELLO, 2001) mostram que há uma grande contradição entre aquilo que se propõe e se recomenda para o ensino da Matemática e aquilo que, de fato, é praticado pelos docentes no cotidiano escolar. Além disso, os professores também desconhecem os processos cognitivos envolvidos na aprendizagem de conteúdos matemáticos e a natureza das dificuldades advindas desse processo, conforme apontado por Rivière (1995). A ausência dessa compreensão tem levado os professores a adotarem procedimentos dogmáticos quanto aos erros dos seus alunos, como afirma Baruk (1996, p. 371): 
Os erros? Se eles deixassem de ser desqualificantes, vergonhosos, para se tornarem objeto de saber para o professor, dinâmica de saber para o aluno que aprenderia quais as lógicas que o levaram a responder como o fez, e qual a lógica à qual essas lógicas, legitimadas e depois recusadas darão lugar, então o sentido começará a circular na aula de matemática, dissipando o clima de angústia, de inércia, de rejeição ou de violência em que vive a maior parte dos alunos.

Diante desse quadro, consideramos importante realizar um estudo para investigar, mais de perto, questões relacionadas à aprendizagem da Matemática no Ensino Fundamental. Para tanto, realizamos uma pesquisa voltada à compreensão dos processos cognitivos envolvidos na resolução dos problemas de natureza multiplicativa, pois, de modo geral, os alunos apresentam dificuldades para trabalhar com as relações proporcionais envolvidas na multiplicação. Para Taxa (2001, p. 5),

[...] o raciocínio multiplicativo não surge facilmente para as crianças em fases de aprendizagem escolar. $\mathrm{Na}$ escola os professores parecem não entender suficientemente os caminhos que as crianças percorrem para entender multiplicações e divisões.

Nesse sentido é importante investir em pesquisas voltadas à aprendizagem desse campo do conhecimento para que melhor possamos compreendê-lo.

\section{A resolução de problemas: estratégia didática e cognitiva}

Um dos grandes problemas do ensino de Matemática diz respeito à falta de relação entre a Matemática praticada no cotidiano e a Matemática ensinada na escola, que privilegia a memorização, a associação e a repetição. Isso, " [ [...] faz com que os alunos quase não consigam reinvestir seus conhecimentos em situações fora do contexto, esquecem com muita facilidade e apresentam enormes dificuldades nos níveis de escolaridade subseqüentes" (FREITAS, 2001, p. 102). As críticas a esses aspectos têm apontado a necessidade de contextualizar os conteúdos a serem trabalhados na escola, de forma a torná-los mais significativos. Nesse sentido, a resolução de problemas tem se mostrado uma das alternativas 
que podem contribuir para melhorar o processo de compreensão dos alunos.

No entanto, apesar de haver desde 1970 investigações sistemáticas sobre resolução de problemas, cujos resultados apontam para a importância de se adequar o ensino a essa metodologia (ONUCHIC; ALLEVATO, 2004), verificamos que ainda é insuficiente o trabalho realizado em sala de aula (GUIMARÃES, 2005).

De acordo com os Parâmetros Curriculares Nacionais - PCN's - de Matemática (BRASIL, 2000), ao colocarmos a resolução de problemas como foco no ensino da Matemática, teríamos uma proposta norteada por alguns princípios, como:

- o ponto de partida da atividade matemática não é a definição, mas o problema;

- o problema não é um exercício em que o aluno aplica, de forma quase mecânica, uma fórmula ou um processo operatório;

- aproximações sucessivas ao conceito são construídas para resolver um certo tipo de problema; num outro momento, o aluno utiliza o que aprendeu para resolver outros;

- o aluno não constrói um conceito em resposta a um problema, mas constrói um campo de conceitos que tomam sentido num campo de problemas;

- a resolução de problemas não é uma atividade para ser desenvolvida em paralelo ou como aplicação da aprendizagem, mas uma orientação para a aprendizagem, pois proporciona o contexto em que se pode apreender conceitos, procedimentos e atitudes matemáticas (BRASIL, 2000, p. 43).

Todos esses princípios também são ressaltados por Onuchic (1999) ao defender a resolução de problemas como metodologia de ensino, afirmando que "[...] o aluno tanto aprende matemática resolvendo problemas como aprende matemática para resolver problemas" (ONUCHIC, 1999, p. 211).

Nesse sentido, os Parâmetros Curriculares Nacionais de Matemática (BRASIL, 2000, p. 44) indicam que para resolver um problema é preciso que o aluno:

- elabore um ou vários procedimentos de resolução(como, por exemplo, realizar simulações, fazer tentativas, formular hipóteses);

- compare seus resultados com os de outros alunos;

- valide seus procedimentos.

Como se pode observar, várias habilidades deverão ser disponibilizadas ao resolver um problema e não simplesmente a capacidade de armazenar informações e aplicá-las mecanicamente, visto que um problema é "[...] qualquer situação que pede uma solução, trazendo aos sujeitos a necessidade de descobrir 
relações e explorá-las, de elaborar hipóteses e verificá-las" (MORO, 1998, p. 7).

Para Ponte e Serrazina (2000, p. 52), a “[...] resolução de um problema constitui um processo de elevado nível de complexidade, que envolve os processos mais simples de representar e relacionar". Representar envolve compreender e usar símbolos, convenções, gráficos e relacionar implica buscar regularidade e generalização entre os conceitos.

Apesar das pesquisas mostrarem que a resolução de problemas é um processo sujeito à elaboração, "[...] os alunos estão acostumados a encontrar a matemática na forma acabada" (HOUSE, 1997, p. 218). Esta imagem é propagada, em muitos casos, pelo próprio professor, que acredita que está ensinando o aluno a resolver problemas, mostrando sua forma de resolução na lousa. Desconhece que a sua forma não pode ser aprendida através da imitação, ficando o desempenho aprendido distante daquele almejado (FIGUEIREDO; GALVÃO, 1999).

Inversamente, ao resolver problemas os alunos mobilizam esquemas e criam estratégias, consideradas pertinentes para cada caso, possibilitando ao professor identificar diferentes raciocínios, dentre eles o multiplicativo, foco deste artigo.

\section{A resolução de problemas multiplicativos}

A multiplicação é uma operação utilizada para realizar contagens sem que seja preciso enumerar os elementos envolvidos. A multiplicação é, no geral, considerada uma das formas mais eficazes de substituir adições com parcelas iguais.

Segundo Granell (1983), o uso do princípio multiplicativo é bastante complexo e envolve domínio de várias relações que ultrapassam a simples identificação da multiplicação como adição de parcelas iguais. Para tanto, a criança precisa compreender dois elementos fundamentais envolvidos no processo da multiplicação: o papel do operador multiplicativo ou o número de conjuntos equivalentes de operações a se realizar e a relação exata entre o número de elementos de cada conjunto e o número de operações que devem ser efetuadas.

Segundo Castro et al. (1996, p. 139-140),

[...] multiplicar é reiterar uma quantidade, em seu nível mais intuitivo. Os dois termos do produto respondem a contextos diferentes; um deles é a quantidade que se repete - multiplicando -, e é um número cardinal concreto, com objetos que se vêem. O outro fator nos diz às vezes que se 
repetem a quantidade inicial - multiplicador-, e é uma espécie de cardinal de segunda ordem ou cardinal de cardinais, muito mais abstrato que o anterior, e por isso mesmo deve ser simbolizado de imediato (tradução nossa).

Nesse sentido, Bryant \& Nunes (1997, p. 142) afirmam que, para compreender a multiplicação e a divisão, é preciso muito mais do que calcular quantidades.

A criança deve aprender e entender um conjunto inteiramente novo de sentidos de números e um novo conjunto de invariáveis, todas as quais estão relacionados à multiplicação e à divisão, mas não à adição e à subtração.

Segundo Martinez (1995), alguns autores têm estudado problemas relacionados ao campo da estrutura multiplicativa, descrevendo-os segundo pontos de vista mais ou menos semelhantes. $\mathrm{O}$ autor apresenta, no quadro a seguir, as classificações de problemas de estrutura multiplicativa mais divulgadas.

\begin{tabular}{|c|c|c|}
\hline Vergnaud (1983, 1988) & Schwartz (1981, 1988) & Nesher (1988) \\
\hline $\begin{array}{c}\text { Isomorfismo de } \\
\text { Medidas }\end{array}$ & I x E = E' & $\begin{array}{c}\text { Regra de } \\
\text { correspondência } \\
\text { ("mapping rule") }\end{array}$ \\
\hline Produto de Medidas & $\begin{array}{c}\text { Comparação } \\
\text { multiplicativa }\end{array}$ \\
\hline
\end{tabular}

QUADRO 1 - CLASSIFICAÇÃO DE PROBLEMAS MULTIPLICATIVOS

FONTE: ADAPTADO DE MARTINEZ (1995, p. 21).

Neste trabalho utilizaremos a classificação apresentada por Vergnaud (1991), que apresenta a multiplicação como pertencente ao campo conceitual das estruturas multiplicativas, cujo tratamento das situações demandam multiplicações e divisões de diferentes tipos, ou à combinação dessas operações.

Para o autor, a complexidade e a diversidade em relação ao domínio das 
relações multiplicativas podem ser ilustradas através da resolução de um conjunto de problemas complexos, classificados como: isomorfismo de medidas, produto de medidas.

- Isomorfismo de medidas: envolve uma relação quaternária, isto é, uma proporção simples entre dois espaços de medida. Os esquemas utilizados para resolver estes problemas envolvem diferentes níveis de dificuldades: multiplicação, regra de três ou divisão. Entretanto, todos podem ser representados por esquemas análogos, onde uma quantidade é procurada, por exemplo: "Tenho 3 pacotes de iogurtes. Existem 4 iogurtes em cada pacote. Quantos iogurtes tenho?" (VERGNAUD, 1991, p. 197).

Em situações envolvendo isomorfismo de medidas, pode-se identificar dois tipos de problemas de divisão: partição e de quotição (medida por quotas). No problema de partição é dada uma quantidade inicial e o número de vezes que esta deve ser distribuída, devendo-se encontrar o valor da unidade, por exemplo: "Paguei R\$ 12,00 por 3 garrafas de vinho. Qual é o preço de uma garrafa?" (VERGNAUD, 1991, p. 198). No problema de quotição (medida por quotas), existe uma quantidade inicial que deve ser dividida em quotas preestabelecidas como mostra o problema: "Pedro tem $\mathrm{R} \$ 12,00$ e quer comprar alguns pacotes de caramelo que custam R $\$ 4,00$ cada pacote. Quantos pacotes ele pode comprar?" (VERGNAUD, 1991, p. 198).

- Produto de medidas: envolve uma relação ternária entre três quantidades, isto é, a composição de dois espaços de medidas em relação a uma terceira medida, tanto no plano numérico como no plano dimensional. Vergnaud (1991) comenta que esta estrutura cartesiana de duas medidas para encontrar uma terceira medida pode ser observada em problemas que envolvem volume, área e combinatória.

No presente trabalho vamos investigar apenas os problemas associados à ideia de combinatória, ou seja, aqueles que envolvem produtos de medidas, cujos enunciados são do tipo: Vou dar uma festa e servirei sanduíches. Para fazer os sanduíches comprei dois tipos de queijo e quatro tipos de pães. Quantos sanduíches diferentes posso servir com um tipo de pão e um tipo de queijo?

Para resolver esse tipo de problema podemos usar procedimentos como uma tabela de dupla entrada ou um diagrama da árvore que permite identificar todos os casos possíveis. Esses problemas podem ser resolvidos por contagem se os números envolvidos forem pequenos, caso contrário o uso do princípio multiplicativo é mais adequado.

Kouba (1989), investigando a resolução de diferentes problemas multiplicativos, descreve uma diversidade de estratégias usadas por alunos do Ensino Fundamental, apontando que os relacionados a produto de medidas foram os mais difíceis para os alunos. 
Um outro estudo realizado por Brito e Correa (2003) faz uma análise do desempenho dos alunos do Ensino Fundamental em problemas de produto de medidas. Os resultados revelam que os alunos em séries mais adiantadas tiveram mais acertos que os demais e que as dificuldades foram mais evidenciadas nos problemas que apresentavam números maiores.

Partindo desses pressupostos, a presente pesquisa pretendeu verificar o desempenho de alunos do $6 .^{\circ}$ ao $9 .^{\circ}$ anos do Ensino Fundamental na resolução de problemas multiplicativos, envolvendo raciocínio combinatório. Com este intuito, realizamos um levantamento das principais estratégias, bem como, dos principais erros produzidos.

\section{Metodologia}

A pesquisa foi realizada em duas escolas públicas (A e B) da cidade de Campo Grande-MS, as quais fizeram parte de uma pesquisa mais ampla realizada anteriormente 6 . Nesta etapa realizamos uma entrevista clínica, com o objetivo de explicitar os processos cognitivos envolvidos na resolução. Para tanto selecionamos, aleatoriamente, 20 alunos de cada escola (dez do $6 .^{\circ}$ e dez do $9 .^{\circ}$ ano). Cada sujeito resolveu individualmente 8 problemas $^{7}$ envolvendo raciocínio combinatório, sendo quatro deles com duas variáveis (problemas de 1 a 4) e quatro com três (problemas de 5 a 8). Houve também uma variação referente ao tamanho dos números utilizados, conforme apresentamos:

1- Vi em uma revista que uma artista de televisão tem 86 pares de sapatos e 54 tipos de meias. Quantas vezes ela pode sair sem repetir a combinação de sapatos e meias?

2- Tatiana vai a uma festa a fantasia usando peruca e óculos. Em uma loja ela encontrou 42 tipos de perucas e 26 tipos diferentes de óculos. De quantas maneiras ela pode se arrumar usando um óculos e uma peruca de cada vez?

3- Uma loja vende bolsas de dois tamanhos (pequenas e grandes) em quatro cores diferentes (preta, marrom, azul e branca). Maria quer comprar uma bolsa nesta loja. Quantos tipos diferentes de bolsa ela pode escolher?

4- Vou dar uma festa e servirei sanduíches. Para fazer os sanduíches com-

6 Em uma fase anterior foi realizada uma pesquisa envolvendo 400 alunos do Ensino Fundamental da rede pública da mesma cidade, na qual foi aplicada uma prova coletiva com 15 problemas de raciocínio combinatório, envolvendo multiplicação e divisão.

7 Foram escolhidos 8 dos 15 problemas aplicados na etapa anterior da pesquisa; os problemas escolhidos foram referentes às situações em que se usava apenas multiplicação. 
prei dois tipos de queijo e quatro tipos de pães. Quantos sanduíches diferentes posso servir com um tipo de pão e um tipo de queijo?

5- Uma sapataria tem 45 pares diferentes de sapatos, 36 tipos de bolsa e 24 tipos de cinto. Cristiane quer comprar um sapato, uma bolsa e um cinto. Quantos conjuntos diferentes de bolsa, sapato e cinto ela pode escolher nesta loja?

6- Valéria tem 32 colares, 92 pulseiras e 65 anéis. De quantas maneiras diferentes ela pode se arrumar, usando apenas um colar, uma pulseira e um anel de cada vez?

7- Em uma sorveteria por quilo existem 6 sabores de sorvete, 3 coberturas e dois tipos de casquinhas. De quantas maneiras diferentes você pode se servir, sabendo que todos os sorvetes são acompanhados de casquinha e cobertura?

8- Na festa de aniversário de Lúcio, cada criança vai receber um saquinho de lembrança. Para receber os saquinhos, a mãe de Lúcio comprou dois sabores de pirulito, 3 sabores de chiclete e 2 sabores de bombons. Quantos tipos diferentes de saquinhos ela pode fazer com um sabor de pirulito, um sabor de chiclete e um sabor de bombom?

A prova foi protocolada em folhas de papel A4, contendo dados relativos à identificação. Ao iniciar a aplicação da prova recomendávamos aos sujeitos que não riscassem ou apagassem as resoluções que considerassem inadequadas, visando manter o registro das tentativas anteriores à solução considerada correta pelos mesmos, pois estas poderiam constituir importantes elementos para análise. Os sujeitos liam os problemas, registravam a resolução e, à medida que eram indagados pela pesquisadora, relatavam as razões dos procedimentos empregados. O diálogo do pesquisador com os sujeitos foi gravado e orientado pelas seguintes questões: Como pensaram para resolver o problema? Qual a pergunta contida no enunciado do problema que deveria ser respondida e qual a resposta encontrada? Como poderiam saber se a resolução apresentada respondia à pergunta do problema e se este permitia uma resolução alternativa.

Com esta metodologia pretendíamos oferecer aos envolvidos a oportunidade de fazer o retrospecto ou a verificação dos seus procedimentos, permitindo que examinassem se a solução obtida estava correta e se existia uma outra maneira de resolver o mesmo problema.

\section{Descrição e análise dos resultados}

Os dados coletados foram transcritos e analisados com duas finalidades: descrever o desempenho dos alunos nos problemas e identificar os procedimentos 
utilizados. Apresentaremos a seguir cada um deles de forma detalhada:

\section{a) Desempenho dos alunos nos problemas}

As informações relativas ao desempenho dos sujeitos das escolas A e B estão dispostas nas Tabelas 1, 2 e 3 apresentadas na sequência.

\section{TABELA 1 - DESEMPENHO DOS ALUNOS NOS PROBLEMAS DE COM- BINATÓRIA REFERENTE À ESCOLA A}

\begin{tabular}{|c|c|c|c|c|c|c|c|c|c|c|c|c|c|c|c|c|c|c|}
\hline \multirow[b]{2}{*}{ Categoria } & \multicolumn{2}{|c|}{$\begin{array}{c}\text { Problema } \\
1\end{array}$} & \multicolumn{2}{|c|}{$\begin{array}{c}\text { Problema } \\
2\end{array}$} & \multicolumn{2}{|c|}{$\begin{array}{c}\text { Problema } \\
3\end{array}$} & \multicolumn{2}{|c|}{$\begin{array}{c}\text { Problema } \\
4\end{array}$} & \multicolumn{2}{|c|}{$\begin{array}{c}\text { Problema } \\
5\end{array}$} & \multicolumn{2}{|c|}{$\begin{array}{c}\text { Problema } \\
6\end{array}$} & \multicolumn{2}{|c|}{$\begin{array}{c}\text { Problema } \\
7\end{array}$} & \multicolumn{2}{|c|}{$\begin{array}{c}\text { Problema } \\
8\end{array}$} & \multirow[t]{2}{*}{ Total } & \multirow[t]{2}{*}{$\%$} \\
\hline & $6^{\circ}$ & $9^{\circ}$ & $6^{\circ}$ & $9^{\circ}$ & $6^{\circ}$ & $9^{\circ}$ & $6^{\circ}$ & $9^{\circ}$ & $6^{\circ}$ & $9^{\circ}$ & $6^{\circ}$ & $9^{\circ}$ & $6^{\circ}$ & $9^{\circ}$ & $6^{\circ}$ & $9^{\circ}$ & & \\
\hline 1 & 1 & 1 & 1 & 1 & 3 & 4 & 4 & 2 & - & 1 & - & - & 2 & 1 & 3 & 1 & 25 & 15,6 \\
\hline 2 & 3 & - & 1 & - & 3 & - & - & - & - & - & - & 1 & 4 & - & 3 & - & 15 & 9,4 \\
\hline 3 & - & - & - & - & - & 2 & - & 1 & - & - & - & - & - & 1 & - & - & 4 & 2,5 \\
\hline 4 & 6 & 7 & 8 & 7 & 3 & 1 & 5 & 4 & 9 & 5 & 10 & 5 & 3 & 4 & 3 & 5 & 85 & 53 \\
\hline 5 & - & 2 & - & 2 & 1 & 3 & 1 & 3 & 1 & 3 & - & 4 & 1 & 4 & 1 & 4 & 30 & 18,8 \\
\hline 6 & - & - & - & - & - & - & - & - & - & 1 & - & - & - & - & - & - & 1 & 0,6 \\
\hline Total & 10 & 10 & 10 & 10 & 10 & 10 & 10 & 10 & 10 & 10 & 10 & 10 & 10 & 10 & 10 & 10 & 160 & 100 \\
\hline
\end{tabular}

1. Acerta operação e resultado

2. Acerta operação e erra resultado

3. Acerta resultado

4. Erra operação e resultado

5. Erra resultado

6. Em branco

É possível verificar na Tabela 1 que a maior porcentagem de respostas dos alunos da escola A foi encontrada na categoria 4, considerando que em 53\% das respostas os alunos usaram procedimentos inadequados para resolverem os problemas, ou seja, escolheram operações que não os conduziriam à resposta correta. Verificamos que $18,8 \%$ das respostas obtidas foram de alunos que não resolveram o problema, apenas apresentaram a resposta de forma incorreta. Podemos notar também que uma pequena porcentagem de respostas $(15,6 \%)$ é proveniente dos alunos que obtiveram êxito, considerando que utilizaram procedimentos próprios de resolução e acertaram o cálculo. Também houve 2,5\% de respostas corretas que não indicavam a operação realizada.

Agrupando as porcentagens das categorias 1 e 3 verificamos que $18,1 \%$ das respostas revelam um bom desempenho dos sujeitos na resolução dos problemas. Observamos, ainda, que $9,4 \%$ das respostas são referentes aos pro- 
cedimentos adequados de resolução, porém com erros nos cálculos. A Tabela 1 nos mostra também que apenas 1 aluno não conseguiu resolver nenhum dos problemas propostos.

A Tabela 2, a seguir, revela o desempenho dos alunos nos Problemas de Combinatória referente à Escola $\mathrm{B}$.

TABELA 2 - DESEMPENHO DOS ALUNOS NOS PROBLEMAS DE COMBINATÓRIA REFERENTE À ESCOLA B

\begin{tabular}{|c|c|c|c|c|c|c|c|c|c|c|c|c|c|c|c|c|c|c|}
\hline \multirow[b]{2}{*}{ Categoria } & \multicolumn{2}{|c|}{$\begin{array}{c}\text { Problema } \\
1 \\
\end{array}$} & \multicolumn{2}{|c|}{$\begin{array}{c}\text { Problema } \\
2 \\
\end{array}$} & \multicolumn{2}{|c|}{$\begin{array}{c}\text { Problema } \\
3 \\
\end{array}$} & \multicolumn{2}{|c|}{$\begin{array}{c}\text { Problema } \\
4\end{array}$} & \multicolumn{2}{|c|}{$\begin{array}{c}\text { Problema } \\
5\end{array}$} & \multicolumn{2}{|c|}{$\begin{array}{c}\text { Problema } \\
6 \\
\end{array}$} & \multicolumn{2}{|c|}{$\begin{array}{c}\text { Problema } \\
7\end{array}$} & \multicolumn{2}{|c|}{$\begin{array}{c}\text { Problema } \\
8\end{array}$} & \multirow[t]{2}{*}{ Total } & \multirow[t]{2}{*}{$\%$} \\
\hline & $6^{\circ}$ & $9^{\circ}$ & $6^{\circ}$ & $9^{\circ}$ & $6^{\circ}$ & $9^{\circ}$ & $6^{\circ}$ & $9^{\circ}$ & $6^{\circ}$ & $9^{\circ}$ & $6^{\circ}$ & $9^{\circ}$ & $6^{\circ}$ & $9^{\circ}$ & $6^{\circ}$ & $9^{\circ}$ & & \\
\hline 1 & - & 1 & - & 1 & 2 & 7 & 3 & 2 & - & - & - & - & - & 3 & - & 3 & 22 & 13,7 \\
\hline 2 & 2 & - & - & - & - & 1 & - & 1 & - & - & - & - & 2 & - & - & - & 6 & 3,7 \\
\hline 3 & - & - & - & - & - & - & - & - & - & - & - & - & 1 & - & - & - & 1 & 0,6 \\
\hline 4 & 8 & 9 & 10 & 9 & 4 & 2 & 5 & 7 & 10 & 9 & 10 & 9 & 6 & 5 & 10 & 4 & 117 & 73 \\
\hline 5 & - & - & - & - & 4 & - & 2 & - & - & 1 & - & 1 & 1 & 1 & - & 1 & 11 & 7 \\
\hline 6 & - & - & - & - & - & - & - & - & - & - & - & - & - & 1 & - & 2 & 3 & 2 \\
\hline Total & 10 & 10 & 10 & 10 & 10 & 10 & 10 & 10 & 10 & 10 & 10 & 10 & 10 & 10 & 10 & 10 & 160 & 100 \\
\hline
\end{tabular}

$\mathbf{N}=\mathbf{1 6 0}$

1. Acerta operação e resultado

2. Acerta operação e erra resultado

3. Acerta resultado

4. Erra operação e resultado

5. Erra resultado

6. Em branco

A Tabela 2 nos mostra um número bastante expressivo de respostas que não revelaram resoluções corretas, ou seja, $73 \%$ apresentaram procedimentos inadequados de resolução e/ou mostraram erros de cálculo. No entanto, 13,7\% das respostas indicam que os sujeitos empregaram procedimentos adequados de resolução e acertaram os cálculos. Houve, ainda, $0,6 \%$ de resultados corretos sem identificação do procedimento utilizado.

Verificamos que $7 \%$ se referem às respostas incorretas e $3,7 \%$ das respostas obtidas foram de sujeitos que, apesar de terem conseguido resolver os problemas de forma correta, erraram o cálculo.

No conjunto das categorias 1 e 3 há um percentual baixo $(14,3 \%)$ de respostas corretas nos problemas apresentados, sinalizando que os problemas de combinatória foram difíceis para os sujeitos, independente do ano cursado.

A Tabela 3, a seguir, aponta a frequência e percentual geral das respostas 
relativas ao desempenho dos alunos nos problemas de combinatória nas escolas A e B.

TABELA 3 - DESEMPENHO GERAL DOS ALUNOS NOS PROBLEMAS DE COMBINATÓRIA NAS ESCOLAS A E B

\begin{tabular}{l|c|c|c|c|c|c|c|c}
\hline & \multicolumn{3}{|c|}{ Escola A } & \multicolumn{3}{c|}{ Escola B } & & \\
\hline Desempenho & $\mathbf{6}^{\mathbf{0}}$ & $\mathbf{9}^{\mathbf{0}}$ & Subtotal & $\mathbf{6}^{\mathbf{0}}$ & $\mathbf{9}^{\mathbf{2}}$ & Subtotal & Total & $\%$ \\
\hline $\mathbf{1}$ & 14 & 11 & $\mathbf{2 5}$ & 5 & 17 & $\mathbf{2 2}$ & $\mathbf{4 7}$ & $\mathbf{1 4 , 7}$ \\
\hline $\mathbf{2}$ & 14 & 1 & $\mathbf{1 5}$ & 4 & 2 & $\mathbf{6}$ & $\mathbf{2 1}$ & $\mathbf{6 , 5}$ \\
\hline $\mathbf{3}$ & - & 4 & $\mathbf{4}$ & 1 & - & $\mathbf{1}$ & $\mathbf{5}$ & $\mathbf{1 , 5}$ \\
\hline $\mathbf{4}$ & 47 & 38 & $\mathbf{8 5}$ & 63 & 54 & $\mathbf{1 1 7}$ & $\mathbf{2 0 2}$ & $\mathbf{6 3}$ \\
\hline $\mathbf{5}$ & 5 & 25 & $\mathbf{3 0}$ & 7 & 4 & $\mathbf{1 1}$ & $\mathbf{4 1}$ & $\mathbf{1 3}$ \\
\hline $\mathbf{6}$ & - & 1 & $\mathbf{1}$ & - & 3 & $\mathbf{3}$ & $\mathbf{4}$ & $\mathbf{1 , 3}$ \\
\hline Total & $\mathbf{8 0}$ & $\mathbf{8 0}$ & $\mathbf{1 6 0}$ & $\mathbf{8 0}$ & $\mathbf{8 0}$ & $\mathbf{1 6 0}$ & $\mathbf{3 2 0}$ & $\mathbf{1 0 0}$ \\
\hline
\end{tabular}

$\mathbf{N}=\mathbf{3 2 0}$

1. Acerta operação e resultado

2. Acerta operação e erra resultado

3. Acerta resultado

4. Erra operação e resultado

5. Erra resultado

6. Em branco

Podemos observar na Tabela 3 que ao agruparmos as categorias que apresentam operação e resultado errados $(63 \%)$ e apenas resultados errados $(13 \%)$ verificamos um percentual elevado de respostas erradas ( $76 \%$ ). O conjunto das respostas evidencia que os problemas de combinatória foram difíceis tanto para os alunos da escola A, quanto para os alunos da escola B, tendo em vista que a diferença entre o desempenho das duas escolas não foi tão grande.

Verificamos que $6,5 \%$ das respostas correspondem aos alunos que apresentaram procedimentos adequados de resolução, porém erraram o cálculo. Destas, $4,6 \%$ foram obtidas na escola $\mathrm{A}$ e as demais na escola B.

A análise do desempenho nos permitiu observar também que nas duas escolas (A e B) houve 1,3\% de respostas provenientes de alunos que não resolveram pelo menos um dos problemas propostos, sendo que a maior parte concentra-se na escola B. 


\section{b) Procedimentos utilizados pelos alunos}

Neste tópico descrevemos e analisamos os dados referentes aos procedimentos empregados pelos quarenta sujeitos das escolas A e B na resolução dos oito problemas propostos. A análise das respostas nos permitiu categorizar quatorze tipos de procedimentos utilizados.

\section{TABELA 4 - PROCEDIMENTOS EMPREGADOS PELOS SUJEITOS DAS ES- COLASAEBNARESOLUÇÃODOS PROBLEMAS PROPOSTOS}

\begin{tabular}{|c|c|c|c|c|c|c|c|c|c|c|c|c|c|c|c|c|c|c|c|c|c|c|c|c|c|c|}
\hline \multirow[b]{4}{*}{ Proc. } & \multicolumn{12}{|c|}{ Problemas com duas variáveis } & \multicolumn{12}{|c|}{ Problemas com três variáveis } & & \\
\hline & \multicolumn{6}{|c|}{ Números grandes } & \multicolumn{6}{|c|}{ Números pequenos } & \multicolumn{6}{|c|}{ Números grandes } & \multicolumn{6}{|c|}{ Números pequenos } & \multirow{2}{*}{\multicolumn{2}{|c|}{$\begin{array}{l}\mathrm{T} \text { o t a } \mathrm{l} \\
\text { Geral }\end{array}$}} \\
\hline & \multicolumn{3}{|c|}{ Prob. 1} & \multicolumn{3}{|c|}{ Prob. 2} & \multicolumn{3}{|c|}{ Prob. 3} & \multicolumn{3}{|c|}{ Prob. 4} & \multicolumn{3}{|c|}{ Prob. 5} & \multicolumn{3}{|c|}{ Prob. 6} & \multicolumn{3}{|c|}{ Prob. 7} & \multicolumn{3}{|c|}{ Prob. 8} & & \\
\hline & A & $\mathrm{B}$ & $\mathbf{T}$ & A & $\mathrm{B}$ & $\mathbf{T}$ & A & B & $\mathbf{T}$ & A & B & $\mathbf{T}$ & A & B & $\mathbf{T}$ & A & $\mathrm{B}$ & $\mathbf{T}$ & A & $\mathrm{B}$ & $\mathbf{T}$ & A & B & $\mathbf{T}$ & $\mathbf{T}$ & $\%$ \\
\hline 1 & 5 & & 5 & 3 & 2 & 5 & 1 & & 1 & & & & 1 & 1 & 2 & 2 & 1 & 3 & & 1 & 1 & & 1 & 1 & 18 & 4,75 \\
\hline 2 & 4 & 3 & 7 & 3 & 5 & 8 & 1 & 1 & 2 & 1 & 1 & 2 & 1 & 1 & 2 & 4 & 5 & 9 & & & & & 1 & 1 & 31 & 8,18 \\
\hline $3 \mathbf{a}$ & 2 & & 2 & 2 & 2 & 4 & 2 & 2 & 4 & 8 & 8 & 16 & 2 & 1 & 3 & 5 & & 5 & 5 & 3 & 8 & & 1 & 1 & 43 & 11,34 \\
\hline $3 b$ & 1 & 1 & 2 & 2 & 2 & 4 & & & & & & & 2 & 1 & 3 & 2 & 1 & 3 & 1 & & 1 & 3 & & 3 & 16 & 4,22 \\
\hline 4 & 9 & 6 & 15 & 9 & 3 & 12 & 1 & & 1 & & & & 3 & & 3 & 5 & 1 & 6 & 1 & 3 & 4 & & & & 41 & 10,81 \\
\hline 5 & & 1 & 1 & & 1 & 1 & & 1 & 1 & & & & 1 & 1 & 2 & & 1 & 1 & & & & & & & 06 & 1,59 \\
\hline 6 & & & & 1 & & 1 & & & & & & & 2 & & 2 & 2 & & 2 & 1 & 1 & 2 & 1 & & 1 & 08 & 2,11 \\
\hline 7 & 2 & 5 & 7 & 2 & 8 & 10 & 1 & 3 & 4 & & 3 & 3 & 3 & 12 & 15 & 3 & 10 & 13 & & 5 & 5 & 4 & 10 & 14 & 71 & 18,73 \\
\hline 8 & 1 & & 1 & 1 & & 1 & 4 & 2 & 6 & 5 & 3 & 8 & & & & 2 & 1 & 3 & 2 & 2 & 4 & 3 & & 3 & 26 & 6,87 \\
\hline 9 & 3 & 2 & 5 & 3 & 1 & 4 & 11 & 9 & 20 & 9 & 3 & 12 & 1 & & 1 & 1 & & 1 & & 3 & 3 & 5 & 3 & 8 & 54 & 14,24 \\
\hline 10 & & 1 & 1 & & 1 & 1 & & & & & 1 & 1 & 2 & 2 & 4 & 2 & & 2 & 3 & & 3 & 3 & 1 & 4 & 16 & 4,22 \\
\hline 11 & & & & & & & & 1 & 1 & & & & & & & & & & & 1 & 1 & & 1 & 1 & 03 & $\mathbf{0 , 8 0}$ \\
\hline 12 & & & & 1 & & 1 & & & & & & & & & & & & & & & & 1 & & 1 & 02 & 0,52 \\
\hline 13 & & 3 & 3 & & 2 & 2 & 1 & 2 & 3 & & 4 & 4 & 3 & 2 & 5 & & 3 & 3 & 1 & 3 & 4 & 1 & 3 & 4 & 28 & 7,39 \\
\hline 14 & 1 & 3 & 4 & & & & & 1 & 1 & & & & 2 & 1 & 3 & 1 & & 1 & 6 & & 6 & 1 & & 1 & 16 & 4,23 \\
\hline
\end{tabular}

Legenda:

Proc $=$ procedimento

Prob $=$ problema

$\mathrm{T}=$ total

$\mathrm{N}=379$

Os procedimentos de 1 a 14 elencados na Tabela 4 referem-se respectivamente às seguintes categorias: Emprega a multiplicação, mas rejeita o procedimento por acreditar que o resultado é muito alto; Indiferenciação entre possibilidades de combinações x possibilidades única (real x possível); Reduz o número de combinações a uma das variáveis do problema, elegendo uma delas 
como resultado ou à quantidade de variáveis do problema; Subtrai as variáveis (raciocínio por correspondência 1 a 1 ) e elege esse resultado como o número de combinações solicitado; Repete os valores das variáveis do problema, indicandoos como resultados isolados; Soma para agrupar as variáveis, depois divide pelo n. ${ }^{\circ}$ de variáveis; Soma o valor das variáveis; Combinações parciais ou assistemáticas, início do princípio multiplicativo; Raciocina por correspondência 1 para muitos; Usa procedimentos impróprios provenientes de outras situações; Associa número por extenso com ausência de dados no problema; Percebe que todos os problemas possuem o mesmo raciocínio; Procedimentos e respostas aleatórios; Raciocina com base em pistas no enunciado.

Observamos na Tabela 4 que o procedimento 7 foi o mais recorrente, ou seja, $18,73 \%$ das menções indicaram que os sujeitos somaram o valor das variáveis para resolver o problema, sendo que houve uma predominância nos problemas com três variáveis. Ao analisarmos a frequência desse procedimento por escola, verificamos que na escola A ele foi mais empregado no problema 5 e na escola B nos problemas 6 e 8 .

O segundo procedimento mais utilizado foi o 9 (14,24\%), no qual os participantes raciocinaram por correspondência um para muitos, principalmente nos problemas 3 e 4 . Observamos que essa frequência foi mais enfatizada no problema 3, em ambas as escolas. Contudo, isso não significa que no decorrer da resolução esse procedimento tenha sido mantido ou proporcionado um resultado correto.

Com 11,34\% das menções aparece o procedimento 3a (reduz o número de combinações a uma das variáveis do problema, elegendo uma delas como resultado). Isso ocorreu especialmente nos problemas 4 e 7 . Em relação às escolas, esse procedimento foi mais frequente no problema 4 , tanto para a escola A como para a escola B.

Outro procedimento utilizado com maior frequência foi o 4 (10,81\%), no qual os alunos subtraíram as variáveis (raciocínio por correspondência 1 a 1) e elegeram o resultado encontrado como o número de combinações solicitado. Observamos que o mesmo foi mais usado nos problemas 1 e 2 (escolas A e B).

Para que possamos melhor evidenciar tais procedimentos, apresentamos na sequência uma descrição dos mesmos, acompanhados de exemplos.

Procedimento 1 - Emprega a multiplicação, mas rejeita o procedimento por acreditar que o resultado é muito alto

As respostas que compõem esta categoria revelam que os sujeitos entrevistados empregaram, inicialmente, a multiplicação para solucionar o problema proposto. No entanto, optaram por substituir essa operação por outra em função 
de acreditarem que o resultado encontrado era muito alto.

Podemos observar que este procedimento aparece com maior frequência nos problemas 1 e 2, os quais são compostos por apenas duas variáveis de valores altos.

Pesquisadora: Por que que você achou que a multiplicação não podia ser?

Pamela: Porque é muita coisa (referindo-se ao resultado encontrado).

Procedimento 2 - Indiferenciação entre possibilidades de combinações x possibilidades única (real x possível)

Neste procedimento percebemos que, em certas ocasiões, os resultados encontrados foram indicados pelos sujeitos como inadequados pelo fato de não distinguirem o possível do viável.

Verificamos principalmente nos problemas 1, 2 e 6 a opção por este procedimento, por serem compostos por variáveis de valores altos.

Pesquisadora: $O$ que significa esse resultado em relação ao problema?

Thaciane: Ela pode usar 81365 de colares, pulseiras e anel de cada vez.

Pesquisadora: Tem outro jeito pra resolver esse problema?

Thaciane: Tem. Usando a conta de menos.

Pesquisadora: Qual forma está correta?

Thaciane: A segunda (Subtração), porque a primeira (Multiplicação) deu muito.

Pesquisadora: Deu muito grande? Não pode ter um número tão grande assim?

Thaciane: Não, porque ela não conseguiria usar [...] 81360 colar, pulseira, anel de cada vez.

Procedimento 3 - Reduz o número de combinações

3a) a uma das variáveis do problema, elegendo uma delas como resultado

Neste caso, os entrevistados escolheram, dentre as variáveis apresentadas no enunciado, apenas uma (a maior ou a menor) e a apontaram como resposta para o problema, sem que qualquer operação fosse adotada. Este procedimento foi utilizado com maior frequência nos problemas 4 e 7. Supomos que isso esteja relacionado ao fato dos enunciados abordarem variáveis de valores pequenos e assuntos próximos do cotidiano (sanduíches e sorvetes).

Pesquisadora: O que significa esse resultado que você encontrou? 
Camila: Significa que ela pode sair 32 vezes sem repetir os pares de sapatos e de meias.

Pesquisadora: Como você chegou a esse resultado?

Paulo: [...] só tem seis sabores diferentes de sorvete, então você pode servir de 6 sabores. [...] pode montar o sorvete de seis formas diferentes.

3b) à quantidade de variáveis do problema

Diferentemente da categoria anterior, nesta, os sujeitos contaram o número de variáveis expostas no problema e o total encontrado foi apontado como resposta.

Evidenciamos uma equidade na distribuição desta categoria quando observamos os dados da Tabela 4. Apesar desse equilíbrio notamos um índice maior nos problemas constituídos por três variáveis.

Pesquisadora: Tá bom Lara, como foi que você chegou a esse resultado?

Lara Beatriz: [...] ela pode colocar um sabor de pirulito, um de chiclete e um de bombom num saquinho e ela pode colocar num outro saquinho outro sabor de pirulito, com dois de chiclete e um bombom.

Procedimento 4 - Subtrai as variáveis (raciocínio por correspondência 1 a 1) e elege esse resultado como o número de combinações solicitado

As menções que compõem esta categoria revelam que a subtração das variáveis foi empregada com a intenção de relacionar uma determinada quantidade de elementos (ex: óculos) à outra (ex: perucas). A diferença encontrada foi assinalada como total de possibilidades.

Esta categoria apareceu com maior ênfase nos problemas 1 e 2, usada algumas vezes como alternativa por aqueles que empregaram o procedimento um (Emprega a multiplicação, mas rejeita o procedimento por acreditar que o resultado é muito alto).

Pesquisadora: O que vocêfez para resolver o problema?

Jonh: Fui subtraindo o número de perucas pelo número de óculos.

Pesquisadora: Mas por que subtração?

Jonh: Porque o número de perucas é maior que os tipos de óculos, para subtrair o maior do menor.

Pesquisadora: Como você descobriu que a conta era essa?

Camila: Somando, quer dizer diminuindo quantos pares de meia ela tem e quantos pares de sapatos. 
Pesquisadora: Você subtraiu por quê?

Camila: Porque ela tem a maioria de sapatos do que de meias.

Procedimento 5 - Repete os valores das variáveis do problema, indicando-os como resultados isolados

Nesta categoria os sujeitos, sem adotar qualquer tipo de cálculo, reproduziram, na íntegra, os valores das variáveis apresentadas nos enunciados e os citaram como resposta do problema.

Pesquisadora: Como você descobriu que esse seria o jeito de resolver?

Paulo: Porque o problema está perguntando quantas bolsas, quantos sapatos e quantos cintos ela pode escolher na loja. Essa loja tem 36 tipos de bolsas, 24 tipos de cintos e 45 sapatos. Ela pode escolher o que a loja tem.

Procedimento 6 - Soma para agrupar as variáveis, depois divide pelo $n .^{\circ}$ de variáveis

Em razão de supor que era preciso calcular a média dos valores revelados no enunciado, esta categoria abarca os sujeitos que formaram, primeiramente, um conjunto a partir da adição desses valores e, em seguida, o dividiram pelo número que correspondia ao total de variáveis.

A Tabela 4 nos mostra um equilíbrio na distribuição deste procedimento, sendo mais presente na maior parte dos problemas com três variáveis $(5,6$ e 7).

Pesquisadora: Qual é o valor?

Tatiana Vicente: [...] somei a quantidade de tudo o que ela tem, deu 189, dividi pelos 3: pelo colar, pela pulseira e pelo anel, que deu 63. Ai o 63 eи peguei [...] eu dividi de novo, que deu 21.

Procedimento 7 - Soma o valor das variáveis

Nesta categoria os sujeitos adicionaram os valores expostos nos enunciados e indicaram como resultado o total encontrado.

Este procedimento foi usado com maior frequência nos problemas 5, $6 \mathrm{e}$ 8 , constituídos por três variáveis.

Pesquisadora: Como você descobriu que a conta tinha que ser esta?

Tatiana Vicente: [...] porque aqui já tá falando que já tem dois tamanhos de quatro cores, o problema tá querendo saber quantos tipos diferentes de bolsa 
ela pode escolher. No caso então, vai ser quatro mais os dois tipos de tamanho que vai ser seis. Então ela pode escolher seis bolsas.

Procedimento 8 -Combinações parciais ou assistemáticas, início do princípio multiplicativo

As respostas que compõem esta categoria revelam que dentre as variáveis apresentadas nos enunciados apenas parte delas foi selecionada no processo de solução do problema, seja mediante o emprego da multiplicação ou de esquemas. Em certos casos, apesar de utilizarem todas as variáveis, somente parte da quantidade de cada uma delas foi considerada.

Os problemas 3 e 4 foram os que apresentaram maior frequência deste procedimento. Cabe ressaltar que os mesmos são compostos por números pequenos.

Pesquisadora: Como você descobriu que esse seria o jeito de resolver o problema 2 ?

Paulo: Para ela usar um óculos e uma peruca ela tem que usar a metade. Foi isso que pensei. Ela vai usar metade desses daqui com a metade desses daqui.

Procedimento 9 -Raciocina por correspondência 1 para muitos

Neste caso, os sujeitos empregaram a multiplicação de todas as variáveis dos problemas, evidenciando a ideia de combinatória.

Este procedimento foi mais recorrente nos problemas 3 e 4, cujos valores das variáveis são pequenos.

Lara: Porque multiplicando os tipos de queijos com os tipos de pães eu vou saber quantos tipos diferentes eu posso servir.

Procedimento 10 - Usa procedimentos impróprios provenientes de outras situações

Neste procedimento os participantes usaram indevidamente estratégias provenientes de outras situações como, por exemplo, equações, para resolver os problemas propostos.

Percebemos o uso deste procedimento especialmente nos problemas que possuem três variáveis $(5,6,7$ e 8$)$.

Pesquisadora: Como dá para resolver o problema 8 ?

Camila: Da mesma forma que eu fiz o exercício 4. Nomear cada um e fazer 
a equação.

Pesquisadora: Como dá para resolver o problema 8?

Paulo: Bom, ela comprou 2 sabores de pirulito, ai eu já coloquei multiplicação e ela colocou mais 3 sabores de chicletes, 2 sabores de bombons. Então coloquei somando. Então eu peguei e fiz a forma distributiva $2(3+2)$ e cheguei nesse resultado.

Procedimento 11 - Associa número por extenso com ausência de dados no problema

Nesta categoria percebemos que, como nem sempre os valores dispostos nos enunciados dos problemas eram formados por algarismos, algumas vezes, os valores não foram selecionados/considerados no momento de resolvê-los.

Pesquisadora: Mas com qual operação você resolve?

Camila: Não tem nenhuma numeração aqui.

Procedimento 12 -Percebe que todos os problemas possuem o mesmo raciocínio

Identificamos apenas dois sujeitos que, independente do fato de terem ou não clareza acerca da forma mais adequada de solucionar os problemas propostos, mencionaram no início (problema 2) ou no final (problema 8) da entrevista que havia algo em comum entre os mesmos.

Pesquisadora: Agora me diz uma coisa Lara, você resolveu todos os problemas por multiplicação por quê?

Lara Lozano: Porque são todos iguais, tem a mesma maneira de resolver.

\section{Procedimento 13 - Procedimentos e respostas aleatórios}

Este procedimento refere-se aos sujeitos que tentaram solucionar um mesmo problema alternando as estratégias, sem estabelecer um critério coerente.

Observamos a adoção deste procedimento principalmente nos problemas $4,5,7$ e 8 .

Pesquisadora: Como você descobriu que tinha que ser essa continha de divisão?

Jacqueline Leite: [...] eu não sei ah, eu olhei aqui e falei: Vou ter que fazer uma divisão!

Pesquisadora: Tem outro jeito de resolver esse problema?

Jacqueline Leite: Tem por mais conta: adição, subtração, multiplicação... 
Pesquisadora: Então, qual é a conta certa?

Jacqueline Leite: Tanto faz!

Procedimento 14 - Raciocina com base em pistas no enunciado

Nesta categoria os sujeitos escolheram as estratégias que utilizariam com base nos termos empregados nos enunciados, principalmente nos problemas 1 e 7, cujos termos serviram como dicas.

Pesquisadora: Como você descobriu que tinha que ter feito uma multiplicação para resolver esse número um?

Laís Barros: Porque aqui só fala em quantas vezes ela pode sair sem a combinação de sapatos, dai eu achei que fosse vezes.

\section{Considerações finais}

Em relação ao desempenho dos sujeitos, fica claro que a resolução de problemas envolvendo combinatória é complexa, pois nas duas escolas houve apenas $16,2 \%$ de respostas corretas (Tabela 3 - categorias 1 e 3). Há ainda que se considerar que o desempenho foi melhor nos problemas com duas variáveis e com fatores de valores baixos (problemas 3 e 4), conforme indicam as Tabelas 1 e 2. Isso talvez possa ser explicado porque os alunos conseguem operar com as variáveis apenas no plano linear, aditivo, como mostra Moro e Soares (2006), ou operam com multiplicação nos casos em que, sendo os números de valores baixos, o cálculo mental ou procedimentos intuitivos podem ser utilizados (TAXA, 2001).

Ao observarmos os procedimentos empregados pelos alunos, fica nítida a dificuldade dos alunos quanto ao raciocínio combinatório, considerando que o princípio multiplicativo foi usado com pouca frequência. Os sujeitos utilizaram, no geral, procedimentos aditivos (juntar os fatores ou subtraí-los), revelando um raciocínio com base na correspondência de um para um, o que mostra uma estratégia primária e natural, segundo apontam algumas pesquisas (BRITO; CORREA, 2003; KOUBA, 1989; MORO; SOARES, 2006).

Quanto à escolaridade, os dados mostraram que não houve diferença de desempenho entre os sujeitos do $6 .^{\circ}$ e do $9 .^{\circ}$ anos, resultado também evidenciado por Moro e Soares (2006).

Embora não tenhamos um controle sobre como os conceitos envolvidos 
nos problemas foram trabalhados pelos professores das escolas envolvidas, sabemos que do ponto de vista da frequência nos materiais didáticos analisados, as situações-problema, envolvendo raciocínio combinatório, foram pouco abordadas (TEIXEIRA et al., 2008).

Em síntese, o exame dos procedimentos adotados pelos sujeitos na resolução dos problemas aqui apresentados deixa claro o quanto a correspondência um para muitos é complexa, sobretudo no caso de problemas com combinatória, e o quanto a sua não compreensão está na raiz das estratégias usadas. Observando a resolução da prova como um todo, algumas dificuldades foram evidenciadas, corroborando os estudos realizados por pesquisadores da área (BRITO; CORREA, 2003; KOUBA, 1989; MORO; SOARES, 2006; TAXA, 2006). Tais dificuldades estão relacionadas aos modelos intuitivos que o aluno tem a respeito da multiplicação (em especial o da soma repetida), os tipos de números (naturais ou decimais) aos quais a operação se aplica; a estrutura semântica do problema; as preferências numéricas quanto ao tamanho dos números, formas de representar o problema e interpretação do enunciado verbal.

No caso deste estudo, podemos elencar algumas dessas dificuldades que, embora nomeadas separadamente, guardam uma estreita relação em si, como relatado a seguir:

1. Dificuldade de diferenciar a correspondência um para um da correspondência um para muitos. Essa dificuldade revela que a criança tenta resolver os problemas usando esquemas próprios da adição e subtração. Ela usa frequentemente um raciocínio aditivo, ou seja, pensa em unir ou separar os elementos ou conjuntos. Esse raciocínio pode ser exemplificado quando ela emparelha os elementos de dois conjuntos e calcula quanto resta (problema 1), quando ela faz a distribuição ou partição do tipo um par de sapato para um par de meia, uma peruca para um óculos, cada tipo de doce para cada saquinho (problemas 1,2 e 8), ou ainda quando ela soma para depois dividir (problemas 5 e 6);

2. Diferença entre contagem e princípio multiplicativo. Mesmo no caso em que a criança usou a matriz, não se pode dizer que ela adotou o princípio multiplicativo, propriamente dito, apenas fez a contagem. Isso ficou evidente nos problemas 3, 4 e 7, nos quais, embora usando a matriz, a leitura que ela faz da mesma não revela a ideia de produto cartesiano. Além disso, a dificuldade de aplicação do princípio multiplicativo ficou mais evidente nos casos em que era preciso multiplicar três fatores entre si (problemas 5, 6, 7 e 8) (TEIXEIRA; VASCONCELLOS; GUIMARÃES, 2009); 
3. A magnitude do produto na multiplicação constituiu uma espécie de obstáculo para a compreensão da natureza dos problemas. Em cada um dos problemas tratava-se de calcular quantas transformações seriam possíveis, dados os fatores apresentados (exemplo: tantos tipos de pães e tantos de queijos, quanto sanduíches possíveis). Ao verificar o resultado encontrado, os sujeitos descartavam o cálculo realizado por ser composto por valores altos (problemas 1, 2 e 6);

4. A relação um para muitos não está expressa no problema, devendo ser construída. Ao tentar estabelecer a relação a criança usa esquemas aditivos, o que a impede de encontrar um produto de medidas, levando a combinações parciais e a confundir as combinações possíveis com um fator do problema, isso ocorreu principalmente no problema 4 (Tabela 4);

5. A relação entre notação simbólica e operação. Essa relação é bastante complicada para a criança, pois na medida em que traduz em notação a operação pensada, o próprio algoritmo induz a interpretações diferentes das verbalizadas inicialmente, como se vê nos problemas 1 e 2 (TEIXEIRA; VASCONCELLOS; GUIMARÃES, 2009).

Verificamos que, em função das limitações decorrentes do modo como a multiplicação é trabalhada na escola, muitas são as dificuldades apresentadas pelos alunos, as quais necessitam ser objeto de maiores investigações, tanto no que se refere aos processos cognitivos, quanto aos procedimentos didáticos.

\section{REFERÊNCIAS}

ANPEPP. Associação Nacional de Pesquisa e Pós-Graduação em Psicologia. X SIMPÓSIO DE PESQUISA E INTERCÂMBIO CIENTÍFICO (24 a 28 de maio) Praia Formosa - Aracruz, ES, 2004.

BARUK, S. Insucesso e matemáticas. Lisboa: Relógio D’Água Editores, 1996.

BRASIL. Ministério da Educação e do Desporto/Secretaria de Educação Fundamental. Parâmetros Curriculares Nacionais. Matemática. Brasília: MEC/SEF, 2000. v. 3. SEF, 1998.

Parâmetros Curriculares Nacionais: Matemática/ $5^{\text {a a }} 8^{\mathrm{a}}$ séries. Brasília: MEC/ 
. Sistema de avaliação da Educação Básica. SAEB, Brasília: MEC/SEF, 2001.

BRITO, M. R. F. de; CORREA, J. O significado do conceito de divisão em crianças de escola elementar. In: III CONFERENCIA ARGENTINA DE EDUCACIÓN MATEMÁTICA. Libro de Resúmenes. Salta: SOAREM/UNSa, 2003. p. 150.

CARNOY, M.; GOVE, A. K.; MARSHALL, J. H. As razões das diferenças de desempenho acadêmico na América Latina: dados qualitativos do Brasil, Chile e Cuba. Revista Brasileira de Estudos Pedagógicos, n. 84, p. 7-33.

CARPENTER, T. P.; MOSER, J. M.; ROMBERG, T. A. (Eds.). Addition and subtraction: A cognitive perspective. Hillsdale, NJ: Lawrence Erlbaum Ass., 1982.

CASTRO, E.; RICO, L.; CASTRO, E. Números y Operaciones. Fundamentos para uma Aritmética Escolar. Madrid: Editorial Síntesis, S.A., 1996.

FIGUEIREDO, R. M. E.; GALVÃO, O. de F. Estratégias de resolução de problemas matemáticos em crianças do ensino fundamental: um estudo descritivo. In: CARMO, J. dos S. Dificuldades da aprendizagem: o instrumento da análise do comportamento no ensino da leitura, escrita e conceitos matemáticos. Belém: UNAMA, 1999.

FRANCHI, A. Situações multiplicativas: diferentes situações e suas inter-relações. In: I SIMPÓSIO BRASILEIRO DE PSICOLOGIA DA EDUCAÇÃO MATEMÁTICA. Anais... Curitiba: Universidade Federal do Paraná, 2002. p. 149-160.

FREITAS, José Luiz Magalhães de. Uma reflexão sobre crenças relativas à aprendizagem matemática. Série-Estudos, Campo Grande: UCDB, n. 11, p. 99-109, 2001.

GOMES, M. G. Solução de problemas de matemática: procedimentos utilizados por sujeitos com graus de escolaridade diferentes. Dissertação (Mestrado em Educação) Faculdade de Educação, Universidade Estadual de Campinas, Campinas, 1998.

GRANELL, C. G. Procesos Cognoscitivos en la aprendizaje de la multiplicación. In: MORENO, M. La pedagogía operatória, un enfoque constuctivista de la educación. Barcelona: Laia, 1983.

HOUSE, P. A. Aventurando-se pelos caminhos da resolução de problemas. In: KRULIK, S.; REYS, R. E. A resolução de problemas na matemática escolar. Tradução de: DOMINGUES, H. H.; CORBO, O. São Paulo: Atual, 1997.

KOUBA, V. L. Children's solution strategies for equivalent set multiplication and division word problems. Journal for Research in Mathematics Education, v. 20, n. 2, p. 147-158, 1989.

LEMOYNE, G.; VINCENT, S.; BRUN, J.; CONNE, F.; PORTUGAIS, J. Addition, addition répetée, multiplication: un trajet éclairé par les schèmes d'action. In: ARTIGUE, 
M.; GRAS, R.; LABORDE, C.; TAVIGNOT, P. (Eds.). Vingt Ans de Didactique des Mathématiques en France. Grenoble: La Pensée Sauvage, 1994. p. 236-242.

MANRIQUE, Ana Lúcia. Processo de formação de professores em Geometria: Mudanças em concepções e práticas. 168f. Tese (Doutorado em Educação: Psicologia da Educação) - PUC/SP, São Paulo, 2003.

MARTINEZ, E. C. Niveles de comprensión en problemas verbales de comparación multiplicativa. Granada: Editorial Comares, 1995.

MEIRA, L. L. Aprendizagem e ensino de funções. In: SCHLIEMANN, A. D.; CARRAHER, D.; SPINILlO, A. G.; MEIRA, L. L.; FALCÃO, J. T. R. Estudos de psicologia da educação matemática. Recife: Editora Universitária da UFPE, 1994. p. 62-84.

MORO, M. L. F. Adição/Subtração: Os caminhos de sua psicogênese na aprendizagem. VII SIMPÓSIO NACIONAL DE PESQUISA E INTERCÂMMBIO CIENTÍFICO. Anais... Gramado, 1998.

MORO, M. L. F.; SOARES, M. T. C. Níveis de raciocínio combinatório e produto cartesiano na escola fundamental. Educação Matemática Pesquisa, v. 8, n. 1, p. 99-124, 2006.

ONUCHIC, L. de la R. Ensino-aprendizagem de matemática através da resolução de problemas. In: BICUDO, M.A. V. (Org.). Pesquisa em educação matemática: concepção e perspectivas. São Paulo: Editora Unesp, 1999.

ONUCHIC, L. de la R.; ALLEVATO, N. S. G. Novas reflexões sobre o ensinoaprendizagem de Matemática através da Resolução de Problemas. In: BICUDO, M. A. V.; BORBA, M. (Org.). Educação matemática: Pesquisa em movimento. São Paulo: Cortez, 2004.

PAVANELLO, Regina Maria. Geometria: Atuação de professores e aprendizagem nas séries iniciais. In: I SIMPÓSIO BRASILEIRO DE PSICOLOGIA DA EDUCAÇÃO MATEMÁTICA. Anais... Curitiba: 2002, p. 172-183.

. O abandono do ensino da Geometria no Brasil: Causas e conseqüências. Zetetiké, Campinas: CEMPEM-FE/UNICAMP, ano 1, n. 1, p. 7-17, 1993.

PONTE, J. P.; SERRAZINA, M. de L. Didática da matemática do $1^{0}$ ciclo. Lisboa: Universidade Aberta, 2000.

RIVIÈRE, A. Problemas e dificuldades na aprendizagem de matemática. Uma perspectiva cognitiva. In: COLL, C. et al. (Orgs.). Desenvolvimento Psicológico e Educação. Necessidades educativas especiais e aprendizagem escolar. Porto Alegre: Artes Médicas. 1995. v. 3. p. 131-156. 
SPINILLO, A. Proporções nas séries iniciais do primeiro grau. In: SCHLIEMANN, A. D.; CARRAHER, D.; SPINILLO, A. G.; MEIRA, L. L.; FALCÃO, J. T. R. Estudos de psicologia da educação matemática. Recife: Editora Universitária da UFPE, 1994. p. 40-61.

TAXA, F. O. S. Problemas multiplicativos e processo de abstração em crianças na $3^{a}$ série do Ensino Fundamental. Tese (Doutorado) - Faculdade de Educação: Universidade Estadual de Campinas, 2001.

. Solução de Problemas com Operações Combinatórias. In: BRITO, M. R. F. (Org.). Solução de Problemas e a Matemática Escolar. Campinas: Editora Alínea, 2006.

TEIXEIRA, L. M. R.; CAMPOS, E. G. J.; VASCONCELLOS, M.; GUIMARÃES, S. D. O uso do livro didático e o desempenho dos alunos do ensino fundamental em problemas de combinatória. Série-Estudos, Campo Grande: UCDB, n. 26, p. 97-112, 2008.

TEIXEIRA, L. M. R.; VASCONCELLOS, M.; GUIMARÃES, S. D. A resolução de problemas multiplicativos de produto de medidas: um caso exemplar. In: BITTAR, M.; MUNIZ, C. A. (Orgs.). A aprendizagem matemática na perspectiva da Teoria dos Campos Conceituais. Curitiba: Editora CRV, 2009. p. 77-93.

VERGNAUD, G. El nino, lãs matemáticas y la realidad. Problemas de la enseñanza de las matemáticas en la escuela primaria. México: Trillas, 1991.

. La théorie des champs conceptuels. Recherche en Didactique de Mathématiques, v. 10, n. 2-3, p. 1333-170, 1990.

. Multiplicative Structures. In: LESH, R.; LANDAU, M. (Orgs.). Acquisition of Mathematics Concepts and Processes. New York: Academic Press, 1983. p. 127-174. 